\title{
La interdisciplinariedad en la investigación aplicada y su impacto en la relación empresa estado y universidad: el caso Occicafé
}

\author{
Rafael Armando Méndez Lozano \\ CEIN Universidad Surcolombiana, Colombia \\ ramendez56@hotmail.com \\ Derly Cibelly Lara Figueroa \\ CEIN Universidad Surcolombiana, Colombia \\ dercibel@gmail.com
}

\section{Surgimiento y evolución de la universidad}

La universidad como establecimiento educativo germina entre mediados del siglo XII y comienzos del siglo XIII en Europa a través de las escuelas catedralicias y las escuelas monásticas, en las que, a un conjunto de maestros, sacerdotes y laicos, la iglesia les confiaba la enseñanza de la doctrina revelada. Desde su nacimiento en la Edad Media bajo circunstancias de dominio de la iglesia, el propósito de la educación se concentró en la formación del clero, caracterizándose por instituirse alrededor de las bibliotecas de las catedrales europeas, su lengua era el latín, los saberes que enseñaban estaban cimentados en la concepción católica del mundo y del hombre, y las facultades, en las que se configuraban los saberes eran: la teología, el derecho y la medicina como las más destacadas y la de artes y filosofía conocidas como facultades menores, en el sentido de que estás se convertían en el primer escalón en el proceso de formación e ingreso a alguna de las facultades mayores.

En su desarrollo las universidades, de acuerdo con Chuaqui (2002), inician su proceso de configuración y expansión cuando se presenta el proceso de separación de las escuelas y los alumnos del poder civil a través de las exenciones que logran de la iglesia y de las autoridades civiles con el estatuto gremial de estudiantes $\mathrm{y}$ maestros, quienes deciden organizarse en asociaciones y profesionales para defender sus intereses, siguiendo el modelo de la universitas.
Resulta oportuno mencionar, que la palabra universidad proviene del latín Universitas, nombre abstracto formado sobre el adjetivo UNIVERSUS-A-UM ("todo", "entero", "universal"). Sin embargo el significado que tiene la palabra en la actualidad empieza a usarse solo hasta fines del siglo XIV puesto que en su origen el término "universidad" era atribuido a una agremiación, sindicato o asociación corporativa que protegía los interesas de las personas dedicadas al oficio del saber y se conocían como las Universitas magistrorum et scholarium, luego respondiendo al proceso de configuración la universidad es denominada universitas litterarum: institución en la que se reúne el saber. Así pues, comprendemos que el modelo universitas simbolizaba un modo de organización en colectivo (Alma mater hispalense, 2015).

Las universidades nacen, entonces, como "expresión del renacimiento intelectual" iniciado entorno a la filosofía y la teología. Conocida como una institución dedicada al intelecto en donde converge todo el saber, la universidad emerge y se desarrolla en medio de circunstancias históricas propias que orientan y condicionan su creación y progreso. Es así como la Universidad de Bolonia, fuerte en Derecho; la de París en Teología y Filosofía; la de Oxford en matemáticas, física y astronomía; y la de Montpellier en Medicina se convierten respectivamente en las primeras instituciones históricas en surgir (Chuaqui, 2002). 
Como producto y creación del ser humano la universita litterarum presenta un conjunto de factores que permiten, además de caracterizarla de acuerdo a una época determinada, vislumbrar su progreso en el tiempo. En ese orden de ideas, haciendo un recorrido, la Universidad del Medioevo de acuerdo con Ortega y Gasset, fue una universidad cultural "dedicada principalmente a la transmisión de la cultura de su época" (Chuaqui, 2002). La cual encuentra precisamente su crisis ante la pérdida de la vigencia de esa síntesis cultural y la exigencia de la sociedad por profesionales y científicos. La investigación para ese momento era desarrollada por personas aisladas e interesadas en un fenómeno sin que existiera alguna estructura organizada referente a la actividad.

Por otro lado, en la primera década del siglo XIX en la época Napoleónica se crea la Universidad Imperial. Orientada a formar intelectuales con un saber práctico útil a la sociedad y caracterizada por asumir "toda la enseñanza, universitaria y escolar, bajo el principio doctrinario de que la función de enseñar las nociones que forman al ciudadano es un privilegio del Estado". (Chuaqui, 2002). Una Universidad Profesionalizante para la cual la enseñanza estaba confiada al que sabía de la disciplina y estuvo nutrida por los avances de la ciencia, sin embargo, el docente, salvo excepciones, no era el mismo investigador. Así pues, la investigación fue incipiente y producida por personas aisladas bajo difíciles circunstancias. Resulta importante precisar que las universidades tradicionales Latinoamericanas siguieron este modelo hasta mediados del siglo XX.

De acuerdo con la revisión histórica realizada por Chuaqui (2002) es posible vislumbrar que en aquella misma década en la que se funda la Universidad Imperial, nace un nuevo género de universidad en Prusia, de notable influencia: La Universidad Humboldtiana. Con un enfoque distinto a la Napoleónica, en la que se le otorga a la investigación el papel principal y sus resultados se incorporan a la enseñanza. Se destaca dentro del modelo de la Universidad Humboldtiana la unidad entre la investigación y la docencia, puesto que el profesor debía ser un investigador y su actividad docente debía consistir en comunicar los nuevos conocimientos y no limitarse a lo que ya estaba escrito. La relación universidad-empresa es otro eje central para este modelo de universidad a través de la investigación aplicada y su transferencia tecnológica.

El modelo Humboldtiano de Universidad permitió el adelanto en el campo científico alemán hasta la II Guerra Mundial. De igual manera influyó en estructuración de la universidad anglosajona, especialmente en la universidad de los Estados Unidos, caracterizada especialmente por desarrollar un sistema de formación educativa eminentemente liberal cuyo propósito es la formación del hombre.

Los modelos de universidad anteriormente descritos se convierten en los tres referentes que influencian el proceso de cambio de la universidad medieval a la universidad moderna y la estructuración de la universidad en el mundo.

Respecto a la universidad latinoamericana y propiamente la colombiana, el modelo napoleónico concebido para dar respuesta a las necesidades del mercado laboral es eminentemente acogido. La universidad colombiana se caracteriza además por ser influenciada en su proceso de configuración por los ideales y hechos políticos y religiosos acontecidos en el desarrollo histórico del país.

La época de modernización de la universidad colombiana, de acuerdo con Soto Arango (2005), se da entre 1957-1970, cuando se desarrolla un plan de modernización de la universidad latinoamericana orientada al mercado del trabajo. Dentro de sus orientaciones se enaltece la investigación como actividad de la educación superior. Sin embargo, sería hasta los 90s producto de la Constitución del 1991 y la reforma de la universidad con la Ley 30 de 1992 que se producen cambios en pro de adaptar y modernizar la universidad en su estructura, organización, modelos educativos para que atendieran el desarrollo científico, tecnológico y de mercado profesional. En consecuencia, 
en el marco de esta comprensión se formula la política científica enmarcada dentro de las demandas del sector productivo, y se introduce la investigación como eje básico para la calidad de la educación universitaria.

En el marco de lo planteado hasta el momento correspondiente al proceso de desarrollo experimentado por la universidad desde su creación hasta nuestros días con la universidad moderna, es plenamente cognoscible vislumbrar como este tipo de organización producto de la actividad del ser humano e inmersa en la estructura social que él configura, ha sido sujeta a grandes transformaciones que la llevaron a comprender la esencia de su quehacer.

Respondiendo al despliegue de lo que comprende el conocimiento, orientado por la razón y sustentado en el método científico, la universidad es reconocida como el lugar donde confluye el saber y el pensar, responsable de producir los grandes descubrimientos que transforman día a día la realidad social y material del ser humano.

La universidad es vista entonces como el lugar donde surgen, o por lo menos se acompaña el desarrollo de las grandes ideas que configuran nuestras vidas a partir de la creación, trasmisión y difusión del conocimiento. Por consiguiente, el ascenso y comprensión de la investigación como eje sustancial que la diferencia de otras instituciones le ha permitido girar su orientación hacia esta actividad como principio rector de su quehacer. De manera que la docencia, como función tradicional -trasmisión-, la proyección social, como la puesta en la realidad del conocimiento generadojunto a la investigación se convierte en las tres dimensiones que se estiman encontrar en una universidad en nuestros días.

En ese orden de ideas, en respuesta a su complejidad como unidad donde converge el saber, y a la incidencia respecto a las múltiples expectativas que se tienen sobre esta, la universidad en el tiempo ha experimentado, además, del proceso de creación y configuración de los campos y áreas del saber, y con ello el nacimiento de nuevas disciplinas, la especialización de estas últimas, así como la creación de herramientas y espacios dedicados a su experticia. Como consecuencia la estructura organizacional para el desempeño de sus funciones, en razón a lo descrito, ha sufrido también continuos ajustes, con la creación de las escuelas, facultades, departamentos, programas, grupos, institutos y centros de investigación, entre otros., desde los que se buscan organizar a la comunidad y responder de manera acertada a los tres frentes previstos como misión (UNESCO, 1998).

Sin embargo, algo ha ocurrido como producto del proceso de configuración de la universidad: El aumento progresivo de la especialización del saber y de su estructura académica, con especial énfasis en la denominada sociedad del conocimiento noción con la que se reconoce en la actualidad a la comunidad global y para la cual el conocimiento ocupa un lugar central en el desarrollo de los procesos culturales, económicos y ecológicos. (UNESCO, 1998).

La producción de conocimiento altamente especializado, que, por supuesto ha sido beneficioso por un lado para el avance de la ciencia y de la tecnología, ha llevado también, al establecimiento artificial del saber a partir de los diferentes métodos, conceptos y comunidades académicas que inciden en la construcción de lenguajes, códigos de verdad, padres, mitos $\mathrm{y}$ sistemas que legitiman las disciplinas $\mathrm{y}$ garantizan su profesionalización. De acuerdo con Uribe Mallarino (2011), solo en 1988 tomando como referencia la nomenclatura internacional estándar de campos de ciencia y tecnología presentada por la UNESCO el saber se clasificaba en " 24 campos de ciencia y tecnología, 249 disciplinas y un número de más de mil de sub-disciplinas" (p157), a partir de las que se estructuran las revistas en las que se socializan los estudios investigativos y se realiza los análisis bibliométricos

En el marco de la especialización del saber, cómo efectos perceptivos en la actualidad, se presentan diversas situaciones similares en todas las universidades que condicionan la producción de conocimiento integral que permita resolver los problemas o fenómenos sociales y materiales 
reales: El trabajo individualista, que predomina en la mayoría de las ocasiones, por parte de los profesores-investigadores quienes producto de su formación e intereses, son expertos en algunas temáticas específicas a las que dirigen todos sus esfuerzos y recursos. Y la perspectiva de trabajo en islas de las facultades, que en algunas ocasiones desdibujan la potencialidad del saber que puede generar como organización una universidad.

En consecuencia, al fortalecimiento del progreso disciplinar, hacia finales de los años sesenta del siglo $X X$ surge un movimiento de científicos y académicos europeos (Uribe Mallarino, 2011, 149) a favor de la inter y multidisciplinariedad como formas de "superar la excesiva especialización del conocimiento", en pro de buscar un abordaje integral a los fenómenos y problemas a partir de la integración del saber. El movimiento de la teoría de los sistemas vivos de Bertalanffy (1968) motiva la necesidad de lograr una visión sistemática y holística del conocimiento, del mismo modo Habermas (1984), filósofo alemán, hace una crítica a la producción utilitaria del saber y a la objetivación del conocimiento que conllevan al abandono de la comunicación inter-subjetiva.

La interdisciplinariedad corresponde a un término que hace referencia a la colaboración de especialistas procedentes de diversas áreas tradicionales, en el campo pedagógico al trabajo científico, en aras por resolver un problema o abordar un fenómeno contemporáneo. En tal colaboración convergen metodologías, conceptos e ideas que permiten generar nuevos conocimientos desde una perspectiva integral a partir de la colaboración o integración de dos o más disciplinas.

Edgar Morín, Basara Nocolescu, Erich Jantsch y Jean Paiget son cuatro académicos que destacan en el marco de su producción teórica, la importancia de la investigación y la generación de conocimiento interdisciplinario. Si bien, encontrar una definición aceptada globalmente sobre la inter (ID) y la transdisciplinariedad que las diferencie, como modo de interacción entre las disciplinas, resulta difícil de encontrar, generalmente se les asocia a descriptores como colaboración, integración, dialogo de saberes, entre otros.

En referencia específicamente a la comprensión de la investigación interdisciplinar como forma de generar conocimiento, para este documento, se tomará aquella que siguiendo a Uribe Mallarino (2012), por el comité interinstitucional que lideró la Academia Nacional de Ciencias de los Estados Unidos y la define como:

Un tipo de investigación realizada por equipos o por individuos por lo cual se integran información, datos, técnicas, herramientas, perspectivas, conceptos y/o teorías de dos o más disciplinas o cuerpos especializados de conocimiento orientados a avanzar en una comprensión fundamental o resolver problemas cuyas soluciones yacen más allá del ámbito de una sola disciplina o área de practica investigativa (NAS, 2005, p. 2). (p.154)

Es ese orden de ideas, hacia la propuesta de construir y producir nuevo conocimiento como esencia de la universidad tanto en el desarrollo del trabajo científico y pedagógico, la interdisciplinariedad resulta necesaria.

Dentro de este proceso de integración del conocimiento conviene destacar la forma de hacer investigación como la vía que permitirá al vehículo -la interdisciplinariedad- llegar a nuevas creaciones. En esta perspectiva resulta la forma en que clasifica la investigación y que generalmente se enmarca en tres variables:

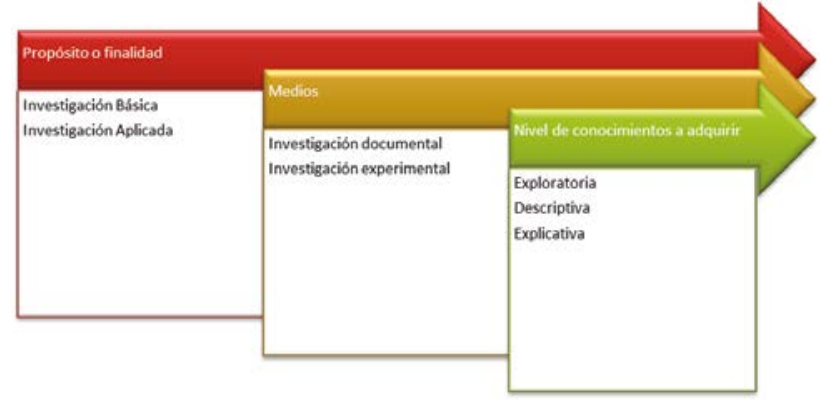

Figura 1. Clasificación de investigación Fuente: Adaptado de Cruz García (2014)

Se encuentra en el centro de las tipologías la Investigación básica y aplicada, ambas son formas 
para entender, verificar, corregir o aplicar el conocimiento. Cada una con finalidades particulares pero interdependientes y necesarias para generar conocimiento integral.
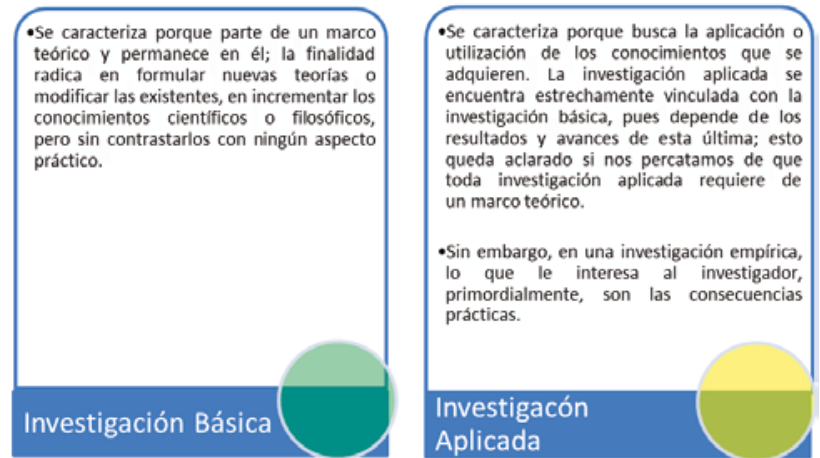

Figura 2. Investigación Básica y Aplicada

Fuente: Adaptado de Cruz García (2014)

La investigación aplicada "busca la generación de conocimiento con aplicación directa a los problemas de la sociedad o el sector productivo" (p 34), se basa en el avance teórico y hallazgos tecnológicos de la investigación básica para generar conocimiento que pueda ser utilizado directamente o a mediano plazo en el mejoramiento de la calidad de vida del hombre (Lozada, 2014). De manera que la investigación aplicada deriva en resultados tangibles.

Si bien está forma de hacer investigación se destaca dentro de las ciencias naturales, de la salud eingeniería y tecnología resulta conveniente subrayar la incidencia de las ciencias sociales en la estructuración, desarrollo y aplicabilidad de este tipo de investigación. Puesto que permite no solo obtener conocimientos sobre la realidad social, sino además dar luces en el estudio y diagnóstico -necesidades y problemas- de una situación social, estructurar herramientas y/o actividades necesarias e identificar posibles efectos de la aplicación de cierto conocimiento, así como de orientar el proceso de aplicación del mismo en la sociedad para que este sea aceptado y apropiado.

En ese orden de ideas las investigaciones básicas y aplicadas son concebidas y desarrolladas desde los campos de las ciencias naturales y las ciencias sociales. Es, entonces, norma implícita como esencia de la ciencia que las investigaciones de distintos tipos, deben apuntar siempre a generar un nuevo valor, sea desde la comprensión, descripción y/o explicación de un fenómeno, llevando, con mayor exigencia en tiempos actuales, a brindar luces en la configuración de elementos tangibles, aplicables que impacten positivamente al mundo -social, ambiental y material- en el que el hombre vive.

\section{La interdisciplinariedad en la Universidad Surcolombiana}

Investigar, actividad connatural en el ser humano garante del proceso de reflexión y creación de conocimiento en el tiempo, como hemos venido describiendo, se convierte en una actividad fundamental en la que la ciencia encuentra su esencia como rama del saber.

La búsqueda de nuevos avances científicos a lo largo del tiempo, ha motivado la configuración de una estructura organizacional a nivel global y nacional que permite su desarrollo. En consecuencia y referente a lo planteado hasta el momento, la universidad es la unidad organizativa cardinal en la que emerge y se despliega la ciencia.

En Colombia la forma en que se erige la actividad investigativa a nivel profesional, se encuentra distribuida en diferentes niveles que reciben orientación por parte del Departamento Administrativo de Ciencia, Tecnología e Innovación COLCIENCIAS, encargado de promover las políticas para la ciencia, la tecnología y la innovación en el país. Las universidades-públicas y privadas- son las organizaciones en donde se inscriben, generalmente, los investigadores que se organizan en: 1) semilleros de investigación como capital semilla en la formación investigativa de los estudiantes de pregrado y 2) grupos de investigación como unidad en la que un conjuntos de docentes-investigadores se reúnen para realizar investigación enfocada a una temática específica.

Así mismo, aparecen en respuesta a la necesidad de integrar el conocimiento $y$ abordar los fenómenos y problemas de una 
manera integral, nuevos actores en la estructura organizativa de la ciencia, en la que se agrupa el trabajo de los grupos de investigación e investigadores en el país.

De acuerdo con COLCIENCIAS dentro de esta estructura se encuentran:

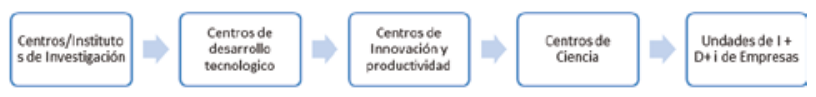

Figura 3. Actores Centros / Institutos de Investigación

Fuente: COLCIENCIAS 2017

Cada actor tiene características particulares normativas y administrativas, pero en esencia con una misión particular: el avance científico y desarrollo de investigaciones básicas y aplicadas que permitan la solución de problemas.

Dentro del grupo de actores sobresalen los centros de investigación de carácter público, privado o mixto, "dedicados a la generación de conocimiento fundamental" (COLCIENCIAS, 2017) en los que convergen, generalmente, diferentes enfoques y disciplinas en la solución de problemas, junto a las unidades o centros de emprendimiento e innovación que buscan en esencia articular la docencia con la investigación y la proyección social, así como promover la cultura del emprendimiento y la innovación en la comunidad universitaria para desarrollar ideas que puedan conducir a emprendimientos inusuales y de alto impacto.

Enestecontexto, laUniversidadSurcolombiana líder en el desarrollo investigativo en la región y en respuesta a las problemáticas y fenómenos naturales y sociales, locales y de orden global, ha venido en los últimos años fortaleciendo su aparato investigativo, a partir del trabajo que ejercen los profesores-investigadores y estudiantes de pregrado y posgrado con la creación de los centros o unidades organizativas, que se convierten en el referente en el que se integra el saber.

En la actualidad la Universidad cuenta con un Centro de Emprendimiento e Innovación que trabaja trasversalmente en la institución e integra el saber enfocado en la aplicación del conocimiento para el desarrollo de emprendimientos empresariales, sociales, culturales o ambientales. También cuenta con cuatro (4) Centros de Investigación en las facultades de Educación, Economía y Administración, Ingeniería y Ciencias Jurídicas y Políticas- en el que se inscriben los grupos de investigación y se asimila el conocimiento científico de la Universidad.

En el marco de esta realidad y reconociendo la naturaleza de la Universidad como institución en la que se reúne el saber y se encarga de producir, distribuir, reproducir y conservar el conocimiento con la intención de generar grandes y positivas transformaciones en la humanidad, se destaca el hacer ciencia e investigar como una de las características esenciales en su quehacer y también, se exige y espera de ella no solo la formación profesionalizante de las personas en la que el desarrollo disciplinar es su única orientación, sino la generación de un saber contextual, aplicable y necesario para impactar el desarrollo de la vida de las personas.

En ese orden de ideas, si bien se ha venido configurando un progreso importante con las actividades que se ejercen desde los semilleros, grupos y centros de investigación a través de la investigación básica y en algunos casos aplicada, resulta necesario integrar el trabajo que realiza toda la estructura organizativa investigativa superando no solo las temáticas y líneas de investigación pertinentes a los programas que conforman las facultades, sino integrando las disciplinas en un accionar interdisciplinario.

Los problemas y fenómenos naturales $\mathrm{y}$ sociales requieren con especial énfasis en razón a la estructura social de nuestros días de soluciones creativas y efectivas basadas en la comprensión y/o descubrimientos con una visión holística, para estudiar y vislumbrar integralmente las diversas dimensiones de una situación o hecho de manera que sea posible lograr avances tangibles, aplicables, y que la impacten directamente la relación universidad - sociedad.

La Universidad Surcolombiana no es ajena a este tipo de exigencias y en cumplimiento 
de su misión debe impactar inicialmente a la región surolombiana. Un caso reciente en el marco del convenio especial de cooperación para el desarrollo de actividades de Ciencia, Tecnología e Innovación No 085 de 2015, es el proyecto "Investigación de las condiciones de control de café especial La Plata Huila, Centro Oriente" a la Asociación de Productores de Café de Alta Calidad OCCICAFÉ, el cual contó con financiación de recursos del Fondo de Ciencia, Tecnología e Innovación FCI del Sistema General de Regalías SGR.

La ejecución del proyecto tenía cinco objetivos orientados a mejorar las condiciones de los pequeños productores asociados y fortalecer la organización:



Figura 4. Esquema con los objetivos generales del proyecto

Fuente: Proyecto de investigación

Para cumplir estos objetivos fue necesario el desarrollo de acciones de articulación e interdisciplinariedad entre el Centro Surcolombiano de Investigación en café CESURCAFÉ y del Centro de Emprendimiento e Innovación CEIN. De esta manera se lograron aportes significativos relacionados con el proceso de beneficio del café (recolección, despulpado, fermentación, lavado y secado), así como propuestas de mejoramiento en procesos de almacenamiento del café seco en finca, transporte, acopio, almacenamiento y seguridad industrial. Complementariamente, se construyó de manera participativa un plan de fortalecimiento de la cultura empresarial y se transfirieron conceptos y experiencias relacionadas con competencias gerenciales.

Esta experiencia dejó en evidencia la posibilidad deabordar demanera integral buenas prácticas y procedimientos fundamentados en la aplicación de conceptos de ingeniería y de carácter organizacional para el fortalecimiento de OCCICAFÉ y sus asociados en un sector tan importante para el departamento como el Cafetero.

Como es de nuestro conocimiento, el café se ha posicionado en el contexto mundial como una de las bebidas preparadas de mayor consumo y genera según la Federación Nacional de Cafeteros, alrededor de US\$200.000 millones de los cuales los agricultores quedan con menos del 10\%. En el mundo del orden de 25 millones de familias depende del café, de los cuales más de 560.000 son familias colombianas, quienes generaron en el 2016 una producción de 14,2 millones de sacos de $60 \mathrm{~kg}$.

Hoy el café del departamento del Huila es reconocido mundialmente por presentar características de cafés especiales, es decir con notas sobresalientes de aroma, fragancia, acidez y cuerpo, que lo definen como un producto con potencial exportador. En este contexto, para el caso de la Asociación de Productores de Café de Alta Calidad del Suroccidente del Huila OCCICAFÉ, se planteó además de la implementación de buenas prácticas y procedimientos para el acopio de cafés especiales, la necesidad de cualificar la organización para construir condiciones que no solo le permitan sobrevivir, sino que también OCCICAFÉ pueda crecer y desarrollarse en sus diferentes áreas operacionales: mercados, finanzas, calidad y talento humano y así mejorar su nivel de productividad y competitividad.

Complementariamente a las labores de transferencia de conocimientos y buenas prácticas y procedimientos para el acopio de cafés especiales, así como de la estructuración del análisis del sector y de un diagnóstico de la organización que facilitó la elaboración de un Plan de Acción con proyectos de corto plazo (1 año), mediano plazo (2,3 años) y largo plazo 
(4,5 años), se generó información útil para otras organizaciones de cafeteros que ha permitido desarrollar publicaciones que además de representar un aporte significativo a los grupos de investigación involucrados, constituyen una evidencia efectiva de la importancia del trabajo articulado entre el Estado, la universidad y las organizaciones y la necesidad de seguir fortaleciendo e impulsando dicha relación.

En ese sentido, tanto para el Centro Surcolombiano de Investigación en Café CESURCAFÉ como para el Centro de Emprendimiento e Innovación CEIN, el desarrollo del proyecto facilitó la integración de la docencia con la investigación y la proyección social, ejemplo de lo que significa una apuesta hacia la construcción de una universidad de tercera generación orientada a la transformación de la realidad como nuestra misión lo reclama.

\section{Referencias bibliográficas}

Alma Mater Hispalense, (2005). De la "universitas" a la "universidad". Disponiblehttps:// personal.us.es/alporu/historia/universitas_ termino.htm.

Apaza Sembinelli, M. (S.F.). Configuraciones y características actuales de la universidad en relación a los modelos tradicionales. Disponible http:// www.feeye.uncu.edu.ar/web/posjornadasinve/ area $1 /$ Politicas $\% 20$ de $\% 20$ educacion $\% 20$ de $\% 20$ evaluacion $\% 20 \mathrm{y} \% 20$ evaluacion $\% 20 \mathrm{de} \% 201 \mathrm{a} \% 20$ politica/221\%20-\%20Apaza\%20-\%20FEEyE.pdf

Bertalanffy, L.v. (1968). General Systems Theory: Foundations Development Aplications. New York. Brasillen.

Chuaqui, B. (2002). Acerca de la historia de las universidades. Rev. chil. pediatr. v.73 n.6 Santiago nov. 2002.http://dx.doi.org/10.4067/S037041062002000600001

COLCIENCIAS (2018). Centros / Institutos de Investigación. Disponible http://www. colciencias.gov.co/portafolio/reconocimiento_ de_actores/centros-institutos-investigacion
COLCIENCIAS (2018). Reconocimiento de actores. Disponible en http://www. colciencias.gov.co/sites/default/files/upload/ reconocimiento/guia-autoevaluacion-centrosinvestigacion-act.pdf

Cruz García, L. (2014). Metodología de Investigación. Disponible en http:// universidadmultitecnica.edu.mx/public/docs/ Material\%20M\%C3\%A9tod os $\% 20$ de $\% 20$ Investigaci\%C3\%B3n.pdf

Habermas, J. (1984). Ciencia y técnica como "ideología". Madrid: Técnos.

Lozada, J. (2014). Investigación Aplicada: Definición, propiedad intelectual e industria. CIENCIAMERICA, $\mathrm{N}^{\circ}$ 3, diciembre 2014, pp (34-39) Universidad Tecnológica Indoamérica

National Geographic, (2013). El nacimiento de la Universidad. Disponible http://www. nationalgeographic.com.es/historia/ grandes-reportajes/el-nacimiento-de-launiversidad_7629/6

Ricardo Gaete Quezada. Historia de la Universidad: Los modelos clásicos. Disponible en http://compartiendoexperienciauniversitaria. blog spot.com.co/2010/07/historia-de-launiversidad-los-modelos.html

Soto Arango, D. (2014). Aproximación histórica a la universidad colombiana. Revista Historia de la Educación Latinoamericana 7, pp. 101-138.

UNESCO, (1998). Declaración mundial sobre la educación superior en el siglo XXI: visión y acción. Disponible en http://www.unesco.org/ education/educprog/wche/declaration_spa.htm

Uribe Mallarino, C. (2012). Interdisciplinariedad en investigación: ¿colaboración, cruce o superación de las disciplinas? Universitas humanística 73, 147-172. 